\title{
Fragments of SLIT3 inhibit cellular migration
}

\author{
THOMAS SCHUBERT ${ }^{1}$, ALEXANDRA E. DENK ${ }^{1}$, ANKE RUEDEL ${ }^{1}$, SIMONE KAUFMANN ${ }^{1}$, \\ ELISABETH HUSTERT ${ }^{2}$, PATRIZIA BASTONE ${ }^{2}$ and ANJA K. BOSSERHOFF ${ }^{1}$ \\ ${ }^{1}$ Institute of Pathology, University of Regensburg, Regensburg; ${ }^{2}$ Scil Technology GmbH, Martinsried, Germany
}

Received May 8, 2012; Accepted July 2, 2012

DOI: 10.3892/ijmm.2012.1098

\begin{abstract}
The repellent factor family of Slit molecules has been described as having a repulsive function in the developing nervous system on growing axons expressing the Roundabout (Robo) receptors. Recent studies determined the effects of Slit molecules on the migratory and invasive potential of several types of tumor cells but also on synovial fibroblasts (SFs) derived from rheumatoid arthritis (RA) patients. To optimize a potential therapeutic application we aimed at generatingfragments of Slit3 showing the same functional ability as the full-length molecule but having the advantage of a smaller size. Recombinant Slit3 proteins were expressed and analyzed by western blotting. Their activity was defined by functional assays such as migration assays with RASF and melanoma cells. Recombinant Slit3 containing only leucine rich repeat domain 2 (D2), the domain important for Robo binding and the minimal functional unit D2 dNC were both able to inhibit migration of RASFs as effectively as Slit3 with all 4 repeats. Collectively, our data showed that the ability of Slit3 to reduce the migratory activity of synovial cells from patients with RA and melanoma cells can be mimicked by small protein fragments derived from Slit3. Slit3 fragments may be helpful in therapeutic attempts; however, further studies are necessary in order to elucidate their activity in vivo.
\end{abstract}

\section{Introduction}

Cellular migration and invasion play major roles in several pathophysiological processes. Tumor cells are known for their aggressive behavior during local invasion and distant metastasis. Furthermore, the destruction of articular structures of the joint, such as cartilage and bone, by synovial fibroblasts

Correspondence to: Professor Anja Katrin Bosserhoff, Institute of Pathology, University of Regensburg, 11 Franz-Josef-Strauss-Allee, D-93053 Regensburg, Germany

E-mail: anja.bosserhoff@klinik.uni-regensburg.de

Abbreviations: SF, synovial fibroblast; RA, rheumatoid arthritis

Key words: repellent factor, Slit, Roundabout, migration, proliferation, rheumatoid arthritis, melanoma, recombinant proteins
(SFs) is a crucial event, particularly in rheumatoid arthritis (RA) $(1,2)$. The 'tumor-like' or 'activated' SFs are localized in the hyperplastic synovium of patients with RA. Supported by adhesion molecules, these rheumatoid arthritis synovial fibroblasts (RASFs) attach to the cartilage, where matrix degrading enzymes released by RASFs finally cause the destruction of the joint.

Repellent factors of the Roundabout (Robo)- and Slitfamily are primarily known to be involved in regulating cell-cell and cell-matrix interactions of migrating cells during embryonic development (3) and by mediating axon guidance through attraction or repulsion of growth cones $(4,5)$. The Robo/Slit system has also been described to mediate cell adhesion of fibroblasts (6) and to induce tumor angiogenesis (7). In previous studies we analysed the role of these factors in melanoma invasion (8) as well as in processes of RASF migration (9), and revealed that Slit3 can potently inhibit cellular migration of both cell types. Further studies, focusing, for example, on other types of cancer, confirmed the strong inhibitory effects of Slit molecules on cellular invasion (10-12).

There are 4 human Robo transmembrane receptors (Robo1, Robo2, Robo3 and Robo4) that share fibronectin type III and immunoglobulin-like domains (Ig), but vary in their cytoplasmatic domains. The ligands for the Robo receptors are the secreted Slit molecules (Slit1, Slit2 and Slit3) consisting of 4 leucine rich repeat domains (D1-4), 7-9 epidermal growth factor (EGF)-like domains, a laminin $\mathrm{G}$ domain and a C-terminal cysteine (Cys)-rich domain (4). Slit binding to Robo receptors is mediated by the second of the 4 highly conserved leucine rich-repeat domains of the ligand Slit and the first extracellular Ig domain of the Robo receptor (13-15). Slit molecules have a molecular weight of approximately $170 \mathrm{kDa}$. Since shorter fragments might be easier and more cost efficient to produce recombinantly, shorter fragments are preferable for functional analysis and therapeutic attempts.

In our study, we concentrated on generating smaller Slit3 fragments, containing the second leucine rich-repeat domain and testing their functionality. We determined whether the Slit3 fragments still show regulatory effects on migration in our 2 model systems, RASF and melanoma cells, and whether these fragments could be used as a pharmaceutical active ingredient to manufacture a pharmaceutical product for the treatment of pathological processes involving cellular invasion. 


\section{Materials and methods}

Cell culture. Synovial tissue samples were obtained during synovectomy and arthroplastic surgery from patients with RA following their informed consent and the approval of the local ethics committee. All RA patients fulfilled the American College of Rheumatology 1987 criteria for the diagnosis of RA. In RA patients, material was sampled from wrist or proximal interphalangeal joints with the joints exhibiting florid synovitis and/or arthritic destruction. Synovial tissue was minced mechanically, washed extensively in sterile phosphate-buffered saline (PBS) and digested with $150 \mathrm{mg} / \mathrm{ml}$ Dispase II (Roche Diagnostics $\mathrm{GmbH}$, Mannheim, Germany) for $1 \mathrm{~h}$ at $37^{\circ} \mathrm{C}$ under continuous agitation. The resulting cell suspension was seeded into tissue culture dishes and cultured in Dulbecco's modified Eagle's medium (DMEM; Gibco-BRL Life Technologies, Basel, Switzerland) containing $10 \%$ fetal calf serum and $100 \mathrm{U} / \mathrm{ml}$ penicillin per $100 \mu \mathrm{g} / \mathrm{ml}$ streptomycin in a humidified atmosphere at $37^{\circ} \mathrm{C}$ followed by the addition of $5 \% \mathrm{CO}_{2}$. In the third passage, adhering fibroblasts were washed, trypsinized and used for RNA isolation or in the assays.

The melanoma cell lines Mel Im and A375 were used as previously described (16). Briefly, cells were maintained in DMEM supplemented with penicillin $(400 \mathrm{U} / \mathrm{ml})$, streptomycin $(50 \mu \mathrm{g} / \mathrm{ml})$, L-glutamine $(300 \mu \mathrm{g} / \mathrm{ml})$ and $10 \%$ fetal calf serum (FCS; Sigma, Deisenhofen, Germany) and split at a 1:5 ratio every 3 days. Cells were detached for subcultivation or assay with $0.05 \%$ trypsin, $0.02 \%$ EDTA in PBS.

Recombinant mouse Slit3 (R\&D Systems, Minneapolis, MN, USA) was used in all cell culture experiments at a concentration of $0.1 \mu \mathrm{g} / \mu \mathrm{l}$.

Cloning and expression of Slit3 fragments. Construct D1-4, including the Slit3 LRR domains 1-4, was amplified using the following primers, 5'-GAC CAT ATG GCC CCT GCC CCA CCA AGT GTA CC-3' and 5'-GAC CCC GGG ATT GCA TTT GGC CAC AAT G-3'; D2, 5'-GAC CAT ATG ATC TCC TGC CCT TCG CCC TGC-3' and 5'-GAC CCC GGG GAA GCA CTC GCT GCT GAA CC-3'; D2 dNC, 5'-GAC CAT ATG ATC GTC GAA ATA CGC CTA GAA C-3' and 5'-GAC CCC GGG TGG GTT TTG GGC TAA GTG GAG-3', and D3, 5'-GAC CAT ATG GAC CTC GTG TGC CCC GAG AAG-3' and 5'-GAC CCC GGG GCT CAG CTG GCA GCT ACT CTC-3'. D2 dNCmut, in which the remaining Cys was exchanged by an alanine (Ala) (Cys85Ala), was cloned by site-directed mutagenesis using the QuikChange ${ }^{\circledR}$ Site-Directed Mutagenesis kit (Stratagene, Amsterdam, The Netherlands) and the primers, 5'-CCA ACA AGA TCA ACG CCC TGC GGG TTA ACA CGT TTC AGG-3' and 5'-CCT GAA ACG TGT TAA CCC GCA GGG CGT TGA TCT TGT TGG-3'. Fragments were cloned into the NdeI and SmaI site of the expression vector pIVEX2.3-MSC and the insert sequences were verified by sequencing. C-terminal HIS tagged SLIT3 proteins were expressed in an RTS 500 system (Roche Molecular Biochemicals, Mannheim, Germany). In vitro transcription and translation were performed according to the manufacturer's instructions as previously described (17). Protein expression was analyzed by western blot analysis (SDS page) using different amounts of protein generated with the RTS 500 expression system.
Protein analysis in vitro (western blotting). RTS-generated proteins were loaded and separated on 12\% SDS-PAGE and subsequently blotted onto a PVDF membrane. After blocking for $1 \mathrm{~h}$ with $3 \%$ BSA/PBS the membrane was incubated for $16 \mathrm{~h}$ with a primary antibody against $6 \mathrm{xHis}(1: 2,000$; Invitrogen Life Technologies, Basel, Switzerland). Then the membrane was washed 3 times in PBS, incubated for $1 \mathrm{~h}$ with an alkaline phosphate-coupled secondary sheep anti-mouse antibody (1:4,000; Chemicon, Hampshire, UK) and then washed again. Finally immunoreactions were visualized by NBT/BCIP (Sigma) staining.

Cloning and expression of Slit 3 fragments in E. coli. Codonoptimized cDNAs of D2, D2 dNCmut and D3 were cloned into the NdeI and NotI site of the expression vector pET22b(+) and the insert sequences were verified by sequencing. C-terminal HIS tagged Slit 3 constructs were expressed in E. coli. For protein isolation $E$. coli cells were treated with lysozyme followed by sonication. Proteins were purified from the supernatant using immobilized metal affinity chromatography (Clontech Laboratories, Mountain View, CA, USA) according to the instructions of the manufacturer. Protein expression was analyzed by standard western blot analysis using an antiHis antibody (Qiagen, Hilden, Germany) and quantified by the Bradford assay.

Migration assay. Migration assays were performed using Boyden Chambers containing polycarbonate filters with $8 \mu \mathrm{m}$ pore size (Costar, Bodenheim, Germany), as previously described (16). Briefly, filters were coated with gelatin. The lower compartment was filled with fibroblast-conditioned medium, used as a chemo-attractant. Synovial fibroblast or melanoma cells, respectively, were harvested with trypsin incubation for $2 \mathrm{~min}$, resuspended in DMEM without FCS at a density of $3 \times 10^{4}$ cells $/ \mathrm{ml}$ and injected in the upper compartment of the chamber on the filter. Following incubation at $37^{\circ} \mathrm{C}$ for $4 \mathrm{~h}$, all cells attached to the upper surface of the membrane were removed. Cells adhering to the lower surface were fixed, stained and counted. Recombinant proteins and peptides were added to the upper chamber of the system. All experiments were repeated at least 3 times.

Statistical analysis. Calculations were performed using the GraphPad Prism software (GraphPad Software, Inc., San Diego, CA, USA). All results are expressed as mean \pm SD (range) or in \%. Error bars represent standard deviation. Comparison between groups was made using the Student's paired t-test (two-tailed).

\section{Results}

Recombinant expression of Slit 3 fragments. Slit treatment revealed a strong negative effect on the migration and cartilage destruction of active RASFs but not on the SFs of healthy donors (9), we therefore speculated about its therapeutic application. Full-length Slit3 would require higher therapeutic doses and might be too expensive for therapy due to their large size. Several publications have previously shown that the second leucine-rich-repeat (LRR2, D2) of Slit2 is necessary for the binding to the Robo receptor (14). In addition, this 


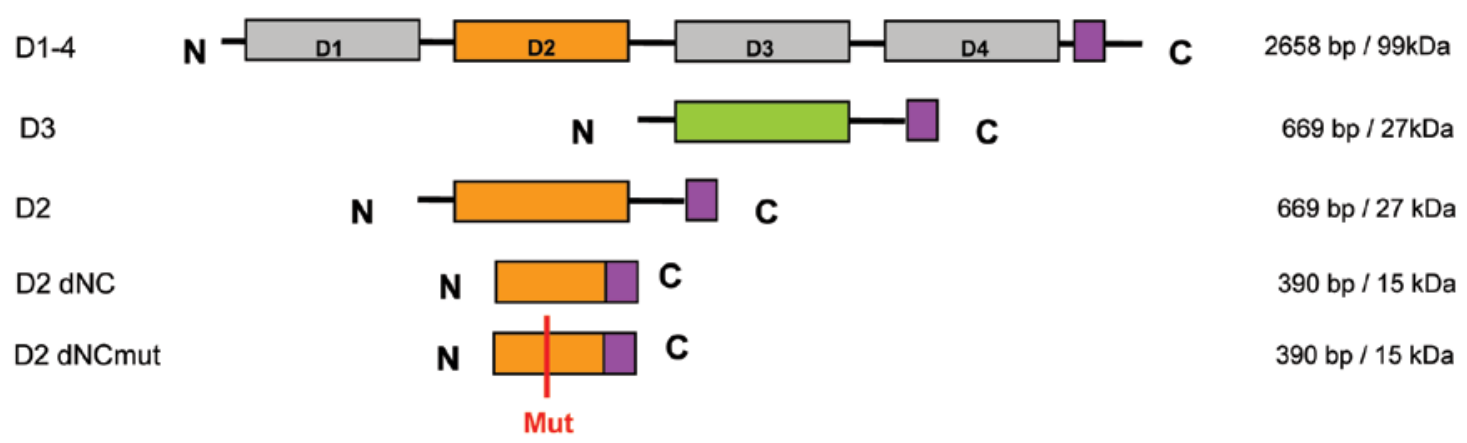

Figure 1. Schematic illustration of recombinant Slit3 fragments. Schematic structure of human Slit3 leucine rich-repeat domains D1-4, D2 and D2 dNC (deleted in $\mathrm{N}$ - and C-terminus). The His-tag is marked as a box.
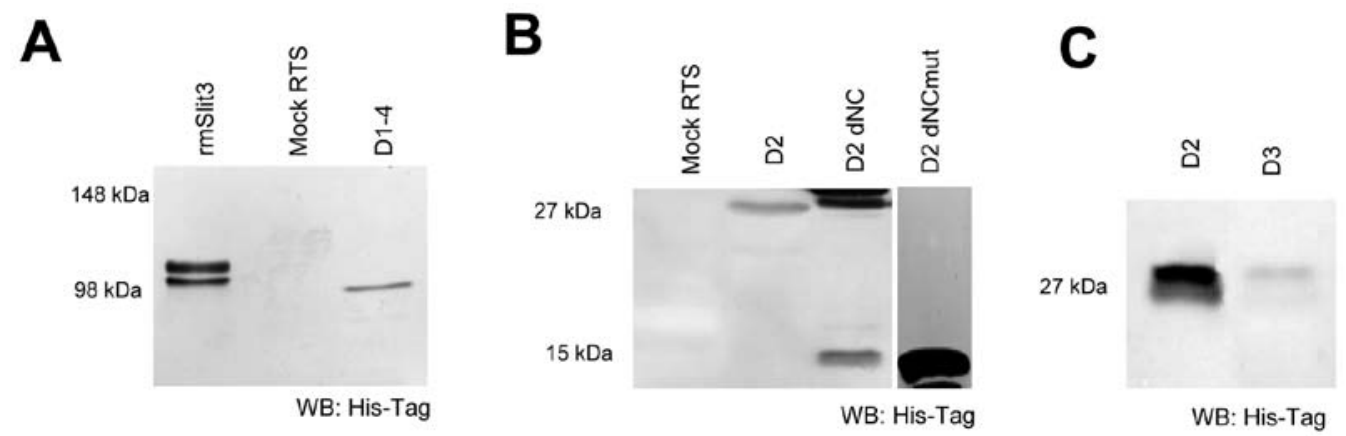

Figure 2. Expression of recombinant Slit3 fragments. (A) rmSlit3 (500 ng), Slit3 D1-4 (0.5 $\mu 1)$, (B) Slit3 D2, D2 dNC and D2 dNCmut (0.5 $\mu 1$ each) and (C) Slit3 D2 and D3 (2 $\mu 1$ each) expressed in the cell-free RTS-System (Roche). Protein expression was analyzed by anti-His-antibody, showing the $99 \mathrm{kDa}$ D1-4 protein, $27 \mathrm{kDa}$ D2 and D3 protein and the $15 \mathrm{kDa}$ D2 $\mathrm{dNC}$ and D2 dNCmut proteins compared to a mock RTS reaction as control. The upper band of the rmSlit3 corresponds to the glycosylated form of Slit3.
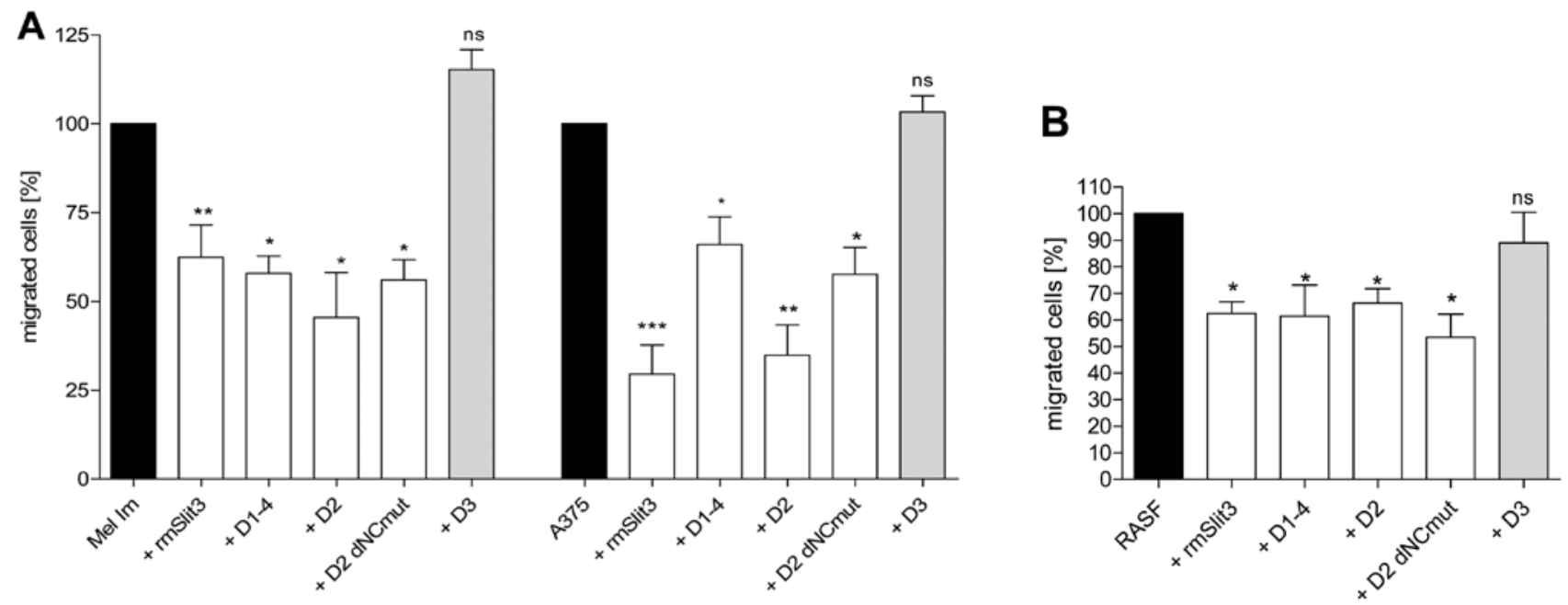

Figure 3. SLIT3 mediated inhibition on the migration. (A) Mel Im, A 375 cells and (B) RASF were treated with recombinant mouse (rm)Slit3, D1-4, D2, D2 dNCmut and the non-receptor-binding D3 fragment of Slit3 in Boyden Chamber migration assays. ns, not significant; ${ }^{*} \mathrm{P}<0.05 ;{ }^{* *} \mathrm{P}<0.01 ;{ }^{* * *} \mathrm{P}<0.001$.

region is not glycosylated enabling recombinant expression in E. coli. To determine whether D2 of Slit3 is sufficient to mediate the inhibition on the migration and destruction by RASF cells and to analyse whether the the $\mathrm{N}$ - and C-terminal region of the D2 domain might be necessary for function and structure, fragments were generated (Fig. 1). We expressed all fragments recombinantly in a transcription/translation system (RTS) and quantified protein amount and protein quality by western blot analysis (Fig. 2). The D2 domain of Slit3 resulted in a protein of $26 \mathrm{kDa}$ (Fig. 2). The shorter, $\mathrm{N}$ - and C-terminally truncated form of D2 (D2 dNC; $15 \mathrm{kDa}$ ) showed, in addition to the expected $15 \mathrm{kDa}$ band, a $30 \mathrm{kDa}$ product as well, possibly occurring by dimerisation of 2 D2 dNC molecules via a free Cys at position 393. To inhibit this 


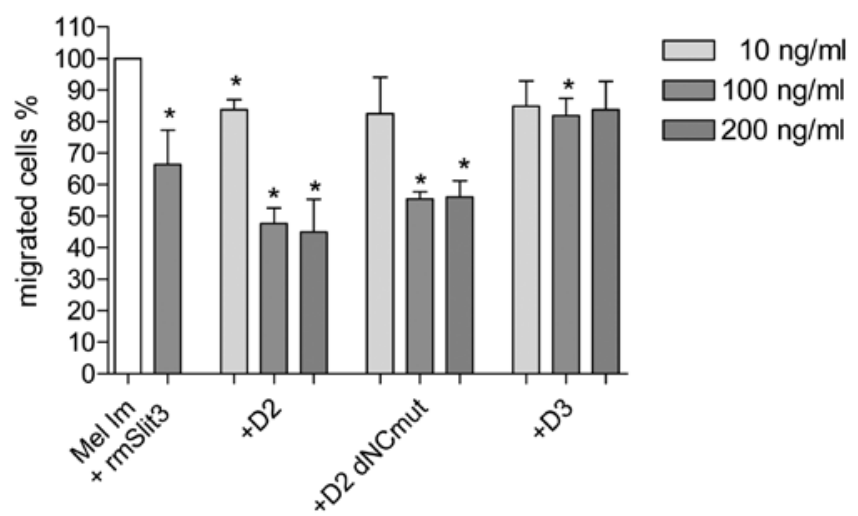

Figure 4. Boyden Chamber Migration Assay of Mel Im treated with D2 and D2 dNCmut. Both Slit3 D2 and Slit3 D2 dNCmut were able to reduce migration of Mel Im $(n=3)$ in early passages compared to untreated control and treatment with the non-receptor-binding fragment D3. Different doses $(10,100$ and $200 \mathrm{ng} / \mathrm{ml})$ were applied. Inhibition by rmSlit3 served as an internal control. $\mathrm{P}<0.05$.

dimerization we mutated the free cysteine (D2 dNCmut) and revealed expression of a $15 \mathrm{kDa}$ construct not able to dimerize (Fig. 2B). This variant was used in further studies instead of D2 dNC. As a control we further expressed the Slit3 D3 domain as this domain is not expected to bind to Robo receptors (Fig. 2C).

Slit3 D2 and D2 dNC inhibit cellular migration of melanoma cells and RASFs. D1-4, D2, D3 and D2 dNCmut were tested in Boyden Chamber Migration Assays on 2 melanoma cell lines (Fig. 3A) and RASFs (Fig. 3B) in comparison to recombinant mouse (rm)Slit3. D1-4, D2 and D2 dNCmut were able to reduce migration of the cells compared to control. Markedly, the D2 dNCmut (Cys85Ala variant) protein which lacks the $\mathrm{N}$ - and $\mathrm{C}$-terminal stabilizing part of the SLIT3 protein sequence inhibits the migration of SFs and melanoma cells indicating that only the minimal binding domain of the protein comprising part of the secondary structure is sufficient for the inhibitory effect. Both, D2 and D2 dNCmut, revealed maximal activity at 100 ng/ml (Fig. 4).

\section{Discussion}

Slits are known to convey repulsive signals via Robo receptors during development and possibly in several pathological conditions. Previous studies demonstrated that Slit can inhibit migration of neuronal cells, leucocytes and glioma cells $(1,7,10)$. We recently showed that Slit3 has a strong inhibitory impact on the migration of aggressive SFs of patients suffering from RA and on melanoma cells in vitro $(8,9)$. The modulation of Slit activity might therefore be a novel therapeutic principle to prevent aggressive cells from destructing healthy tissue in completely different groups of diseases such as rheumatic diseases or cancer. The current RA therapy is mainly based on anti-inflammatory agents such as glucocorticoids, non-steroidal anti-rheumatic drugs (NSAIDs), diseasemodifying anti-rheumatic drugs (DMARDs) and in more recent approaches on modulating the immune system (18).
Malignant melanoma is usually treated by surgical excision complemented by additional therapies such as chemotherapy, radiation therapy or immunotherapy depending on tumor stage $(19,20)$. Chemotherapy, immunotherapy or anti-inflammatory drugs in the treatment of RA or malignant melanoma can frequently have adverse effects ranging from mild to more serious conditions. The inhibition of migration of aggressive synovial cells or melanoma cells using Slit3 or fragments of Slit3 might be a less damaging approach to treat RA or malignant melanomas. Since cell proliferation of RASF cells was weakly suppressed by Slit3 (9) mainly migrating cells should theoretically be affected by therapeutic intervention. Cell migration of constituting cells in target tissues such as the dermal cells of the skin or different cell types in joint tissue is not of major importance for tissue homeostasis. Possible mechanisms for the induction of side-effects may therefore be limited.

Full-length Slit or Slit fragments containing leucin-rich repeat domains D1-4 (7) may not be appropriate for therapeutic use. Due to their large size they are difficult to express recombinantly, and may be unstable and also difficult to apply in vivo. Since several publications demonstrated the smaller second domain of Slit2 D2 to be the domain necessary for Robo binding $(21,22)$, we analyzed the structure of this domain closely and hypothesized that the $\mathrm{N}$ - and C-terminal region may not be necessary for Robo binding. Several Slit3 fragments of the D2 domain were designed and recombinantly expressed including truncated and mutated fragments (Fig. 1). We analyzed the effects of these fragments on SFs of RA patients and malignant melanoma cells in vitro in comparison to rmSlit3.

Using Boyden Chamber migration assays, we showed that the second domain of Slit3 (D2) is sufficient to exhibit inhibitory activity towards RASF and melanoma cell migration as effectively as recombinant Slit3 containing all 4 leucine rich repeats (Fig. 3). Also, the truncated form of D2 (D2 dNC) which lacks the $\mathrm{N}$ - and C-terminal part of the domain as well as its mutated variant is adequate to inhibit migration of RASFs and melanoma cells (Fig. 3). At doses of $100 \mathrm{ng} / \mathrm{ml}$ both fragments D2 and D2 dNCmut were able to reduce migration of melanoma cells to approximately $50 \%$ in the Boyden Chamber (Fig. 4). This means that Slit3 fragments are sufficient to exert the inhibitory effect of Slit3 via the Robo-receptor. Local application of Slit3 fragments into the joint cavity of patients suffering from RA may therefore be a feasible way to prevent joint destruction by aggressive SFs. Fortunately, the D2 domain is not glycosylated enabling recombinant expression in E. coli.

Taking into account the in vitro data reported here, peptides derived from Slit3 may be a powerful therapeutic tool to decelerate or even prevent tissue destruction by aggressive cells in several pathological conditions.

\section{Acknowledgements}

This study was supported by the Wilhelm Sander Foundation and the German Research Foundation (DFG), P.B. and E.H. are employees of the Scil Technology GmbH. A.B. and T.S. have a cooperation agreement with the Scil Technology $\mathrm{GmbH}$. 


\section{References}

1. Guan CB, Xu HT, Jin M, Yuan XB and Poo MM: Long-range $\mathrm{Ca}^{2+}$ signaling from growth cone to soma mediates reversal of neuronal migration induced by slit-2. Cell 129: 385-395, 2007.

2. Yasuda T: Cartilage destruction by matrix degradation products Mod Rheumatol 16: 197-205, 2006.

3. Alten R, Gromnica-Ihle E, Pohl C, Emmerich J, Steffgen J, Roscher R, Sigmund R, Schmolke B and Steinmann G: Inhibition of leukotriene B4-induced CD11B/CD18 (Mac-1) expression by BIIL 284, a new long acting LTB4 receptor antagonist, in patients with rheumatoid arthritis. Ann Rheum Dis 63: 170-176, 2004.

4. Dickson BJ and Gilestro GF: Regulation of commissural axon pathfinding by slit and its Robo receptors. Annu Rev Cell Dev Biol 22: 651-675, 2006.

5. Chen JH, Wen L, Dupuis S, Wu JY and Rao Y: The N-terminal leucine-rich regions in Slit are sufficient to repel olfactory bulb axons and subventricular zone neurons. J Neurosci 21: 1548-1556, 2001.

6. Krasnokutsky S, Samuels J and Abramson SB: Osteoarthritis in 2007. Bull NYU Hosp Jt Dis 65: 222-228, 2007.

7. Wu JY, Feng L, Park HT, Havlioglu N, Wen L, Tang H, Bacon KB, Jiang Z, Zhang X and Rao Y: The neuronal repellent Slit inhibits leukocyte chemotaxis induced by chemotactic factors. Nature 410: 948-952, 2001.

8. Denk AE, Braig S, Schubert T and Bosserhoff AK: Slit3 inhibits activator protein 1-mediated migration of malignant melanoma cells. Int J Mol Med 28: 721-726, 2011.

9. Denk AE, Kaufmann S, Stark K, Schedel J, Lowin T, Schubert T and Bosserhoff AK: Slit3 inhibits Robo3-induced invasion of synovial fibroblasts in rheumatoid arthritis. Arthritis Res Ther 12: R45, 2010.

10. Mertsch S, Schmitz N, Jeibmann A, Geng JG, Paulus W and Senner V: Slit2 involvement in glioma cell migration is mediated by Robol receptor. J Neurooncol 87: 1-7, 2008.

11. Stella MC, Trusolino L and Comoglio PM: The Slit/Robo system suppresses hepatocyte growth factor-dependent invasion and morphogenesis. Mol Biol Cell 20: 642-657, 2009.
12. Kim HK, Zhang H, Li H, Wu TT, Swisher S, He D, Wu L, Xu J, Elmets CA, Athar M, et al: Slit2 inhibits growth and metastasis of fibrosarcoma and squamous cell carcinoma. Neoplasia 10: $1411-1420,2008$

13. Hohenester E, Hussain S and Howitt JA: Interaction of the guidance molecule Slit with cellular receptors. Biochem Soc Trans 34: 418-421, 2006.

14. Morlot C, Thielens NM, Ravelli RB, Hemrika W, Romijn RA, Gros P, Cusack S and McCarthy AA: Structural insights into the Slit-Robo complex. Proc Natl Acad Sci USA 104: 14923-14928, 2007.

15. Muller-Ladner U, Ospelt C, Gay S, Distler O and Pap T: Cells of the synovium in rheumatoid arthritis. Synovial fibroblasts. Arthritis Res Ther 9: 223, 2007.

16. Denk AE, Bettstetter M, Wild PJ, Hoek K, Bataille F, Dietmaier $\mathrm{W}$ and Bosserhoff $\mathrm{AK}$ : Loss of maspin expression contributes to a more invasive potential in malignant melanoma. Pigment Cell Res 20: 112-119, 2007.

17. Stoll R, Lodermeyer S and Bosserhoff AK: Detailed analysis of MIA protein by mutagenesis. Biol Chem 387: 1601-1606, 2006.

18. Majithia V and Geraci SA: Rheumatoid arthritis: diagnosis and management. Am J Med 120: 936-939, 2007.

19. Mouawad R, Sebert M, Michels J, Bloch J, Spano JP and Khayat D: Treatment for metastatic malignant melanoma: old drugs and new strategies. Crit Rev Oncol Hematol 74: 27-39, 2010.

20. Atallah E and Flaherty L: Treatment of metastatic malignant melanoma. Curr Treat Options Oncol 6: 185-193, 2005.

21. Rajagopalan S, Nicolas E, Vivancos V, Berger J and Dickson BJ: Crossing the midline: roles and regulation of Robo receptors. Neuron 28: 767-777, 2000.

22. Nguyen Ba-Charvet KT, Brose K, Marillat V, Kidd T, Goodman CS, Tessier-Lavigne M, Sotelo $\mathrm{C}$ and Chedotal A: Slit2-Mediated chemorepulsion and collapse of developing forebrain axons. Neuron 22: 463-473, 1999. 